Supporting Information

\title{
A microporous polymer with suspended cations for anion exchange membrane fuel
}

\section{cells}

Ziqin Lia,b, Jing Guo , Jifu Zheng ${ }^{a^{*}}$, Tauqir A. Sherazic, Shenghai Lia, $b^{*}$, Suobo Zhang ${ }^{a, b}$

a Key Laboratory of Polymer Ecomaterials, Changchun Institute of Applied

Chemistry, Chinese Academy of Sciences, Changchun, 130022, China.

b University of Science and Technology of China, Hefei, 230026, China.

c Department of Chemistry, COMSATS University Islamabad, Abbottabad

Campus, Abbottabad, 22060, Pakistan.

Synthesis

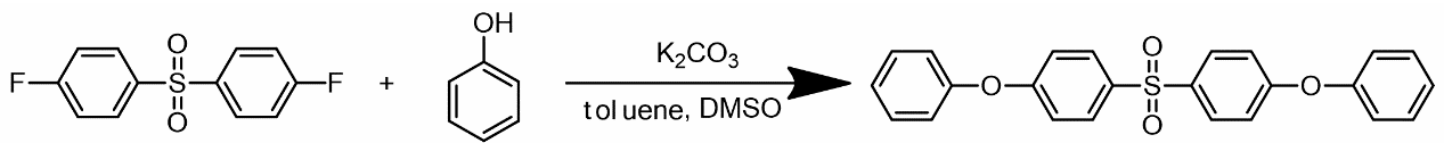

Scheme S1. The synthetic route of AES.

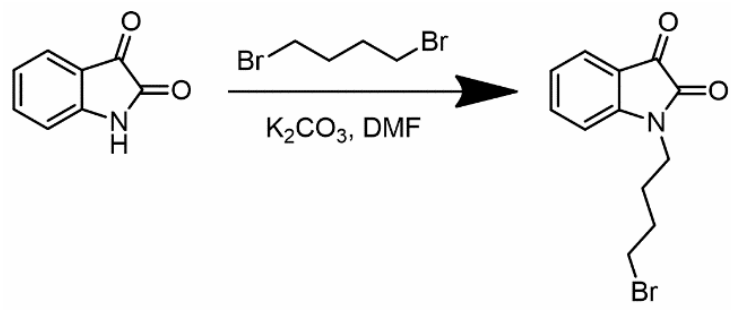

Scheme S2. The synthetic route of BID. 


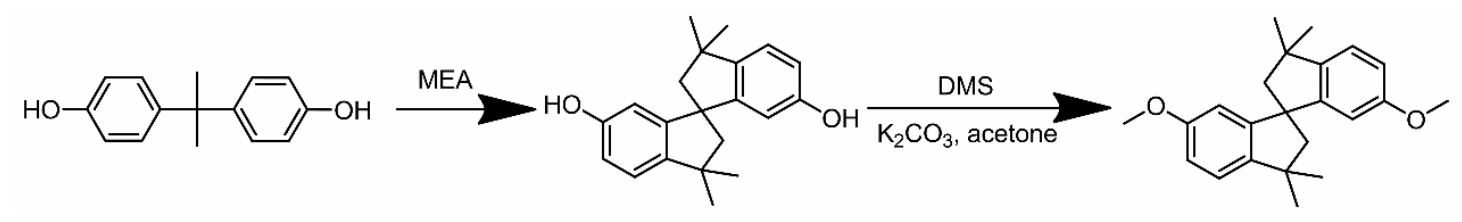

Scheme S3. The synthetic route of SBP and SBI.

4,4'-sulfonylbis(phenoxybenzene) (AES). The synthesis of AES is shown in Scheme S1. $50 \mathrm{~g}$ of phenol was dissolved in $150 \mathrm{~mL}$ DMSO and added to a three-necked flask equipped with Dean-Stark trap, condenser tube, and a nitrogen supply tube. $73.3 \mathrm{~g}$ of potassium carbonate and $100 \mathrm{~mL}$ of toluene were fed, followed by nitrogen purge, dehydration at $150{ }^{\circ} \mathrm{C}$, and toluene was removed by heating. Then 4,4'-difluorodiphenyl sulfone was added and reaction was performed at $165^{\circ} \mathrm{C}$. for 2 hours. The resulting material was settled into water, washing with water and ethanol several times and dried to obtain white powder.

1-(4-bromobutyl)indoline-2,3-dione (BID). To a $500 \mathrm{~mL}$ three-necked flask, isatin $(30 \mathrm{~g})$ and anhydrous DMF $(200 \mathrm{~mL})$ were added. After isatin was dissolved, $\mathrm{K}_{2} \mathrm{CO}_{3}(56.39 \mathrm{~g})$ was added to the solution with stirring, and stirring was continued for 30 minutes at room temperature. Then 1, 4-dibromobutane (121 $\mathrm{mL}$ ) was slowly dripped through a constant pressure dropping funnel and the reaction was performed at room temperature. The completion degree of the reaction was monitored by TLC. After filtration to remove $\mathrm{K}_{2} \mathrm{CO}_{3}$, excess 1, 4dibromobutane was distilled off under reduced pressure. The red needle crystal product was further purified by recrystallization (ethanol as solvent). The yield was $80 \%$. 
6,6'-dihydroxy-3,3,3',3'-tetramethyl-1,1'-spirobiindane (SBP). SBP was synthesized via acid-catalyzed reactions from the starting material bisphenol $\mathrm{A}$ according to the previous literature. (1) The synthesis method of SBP is shown in Scheme 1. Firstly, $200 \mathrm{~g}$ of bisphenol A was melted at $140{ }^{\circ} \mathrm{C}$, and then $10 \mathrm{~g}$ of methanesulfonic acid was added. After the mixture was reacted with mechanical stirring for 5 hours, it was slowly poured into ice water. The precipitated solid was washed repeatedly with water and recrystallized with ethanol as a solvent, thereby obtaining a white flocculent product $(30 \mathrm{~g})$.

6,6'-dimethoxy-3,3,3',3'-tetramethyl-1,1'-spirobisindane (SBI). SBI is derived from the methylation of SBP, (2) as shown in Scheme S3. $20 \mathrm{~g}$ of SBP was dissolved in $160 \mathrm{~mL}$ of acetone. In an ice bath, $45 \mathrm{~g}$ of potassium carbonate was added and stirred for 15 min. Dimethyl sulfate was gradually added dropwise to the mixture and reacted at room temperature for 10 hours. Then the system was poured into ice water and continuously stirred. The white precipitate was filtered, washed with water several times, and then recrystallized with petroleum ether.

\section{Figures}



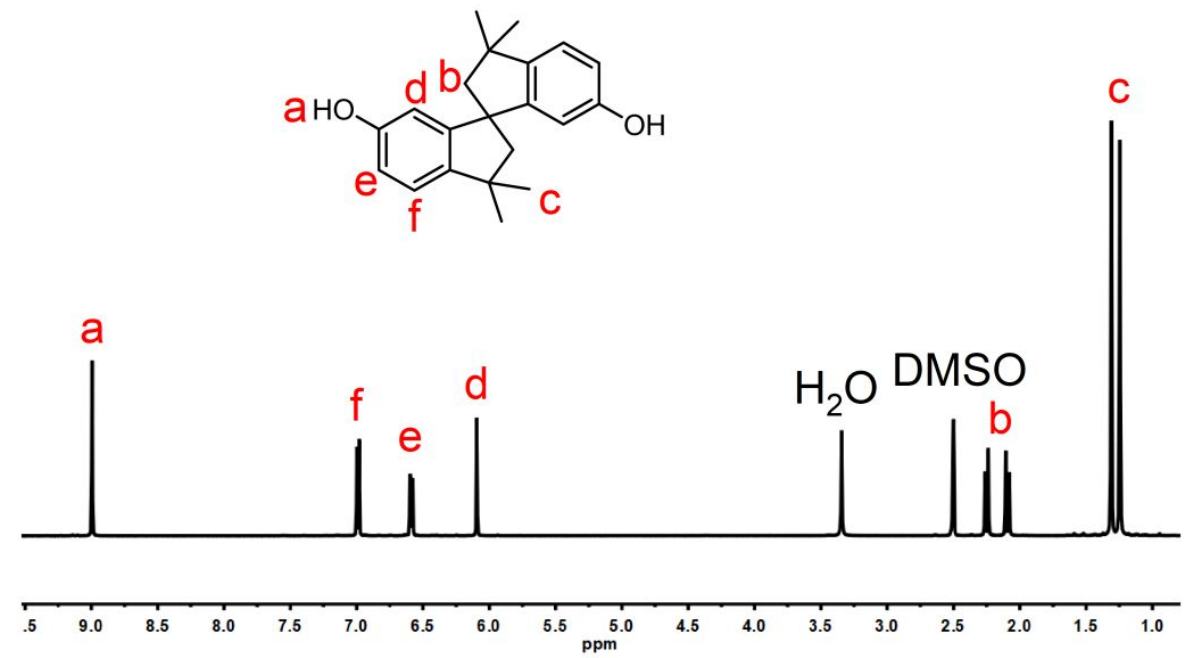

Figure S1. ${ }^{1} \mathrm{H}$ NMR spectra of 6,6'-dihydroxy-3,3,3',3'-tetramethyl-1,1'spirobiindane (SBP).

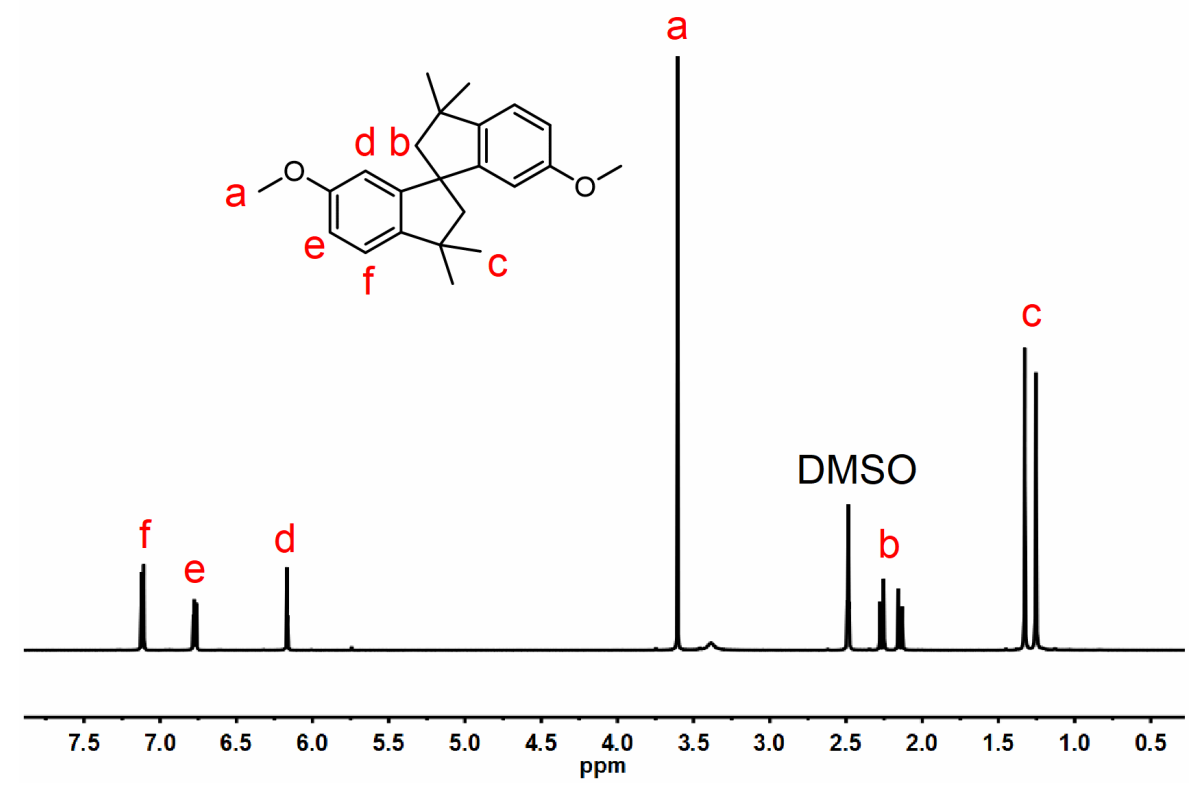

Figure S2. ${ }^{1} \mathrm{H}$ NMR spectra of $6,6^{\prime}$-dimethoxy-3,3,3',3'-tetramethyl-1,1'spirobisindane (SBI). 

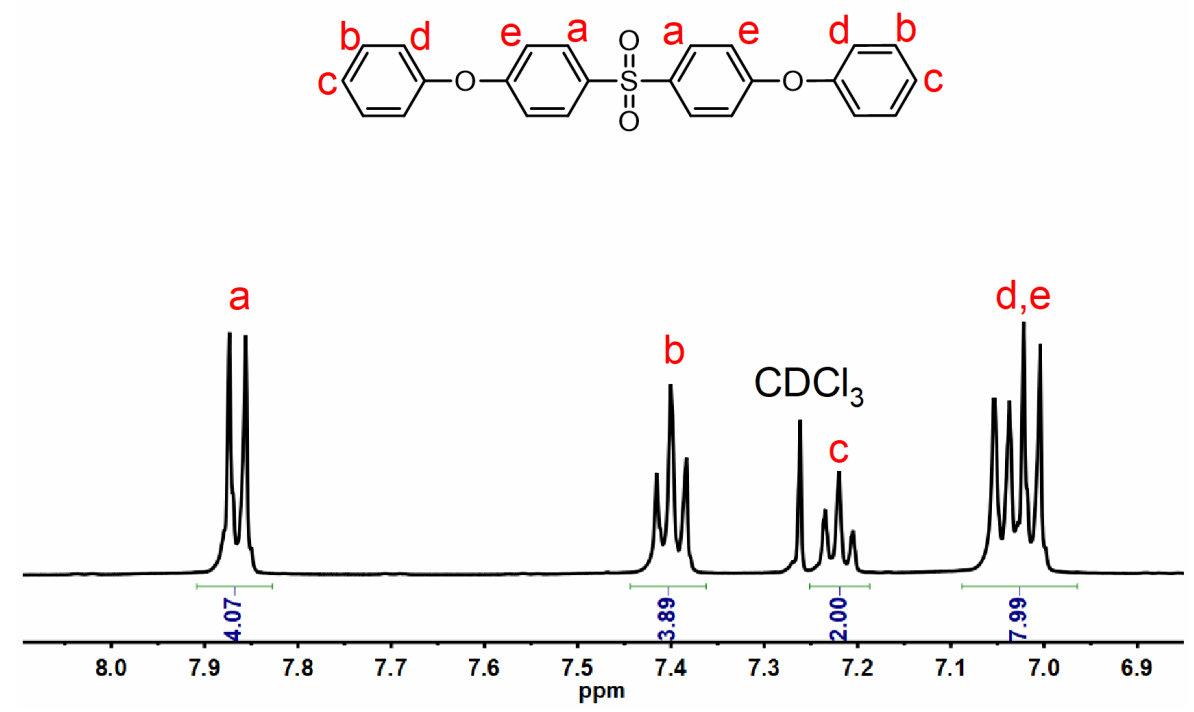

Figure S3. ${ }^{1} \mathrm{H}$ NMR spectra of 4,4'-sulfonylbis(phenoxybenzene) (AES).

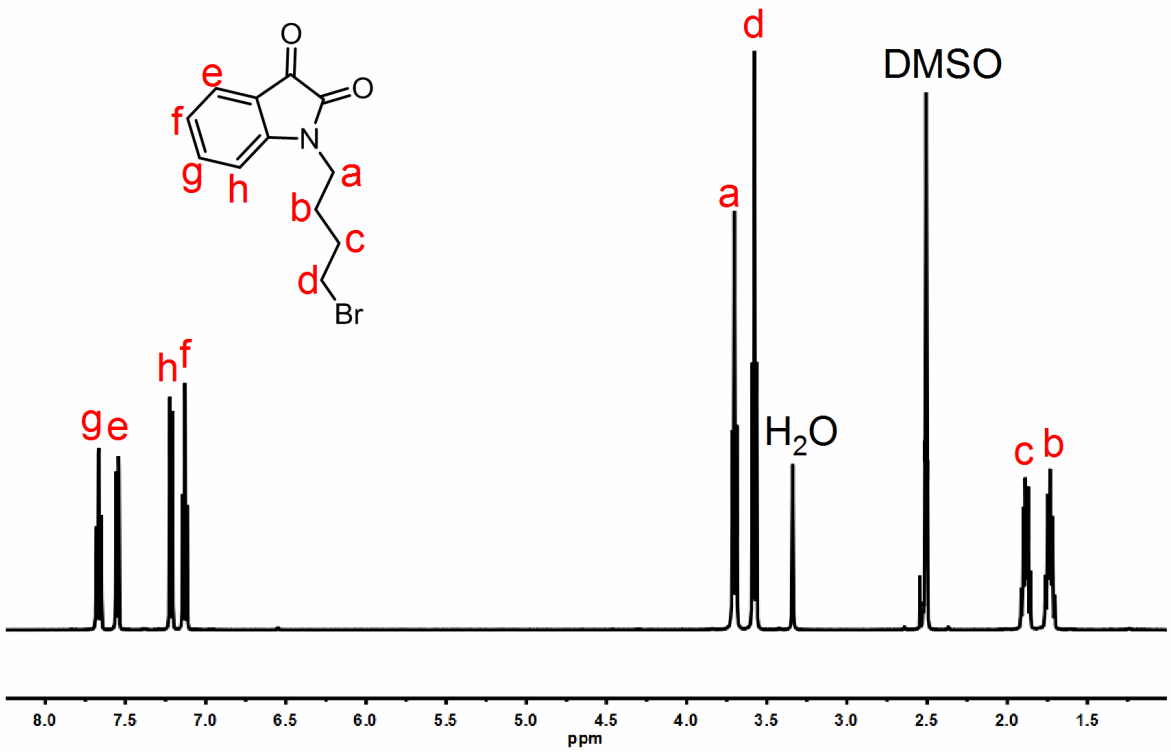

Figure S4. ${ }^{1} \mathrm{H}$ NMR spectra of 1-(4-bromobutyl)indoline-2,3-dione (BID). 

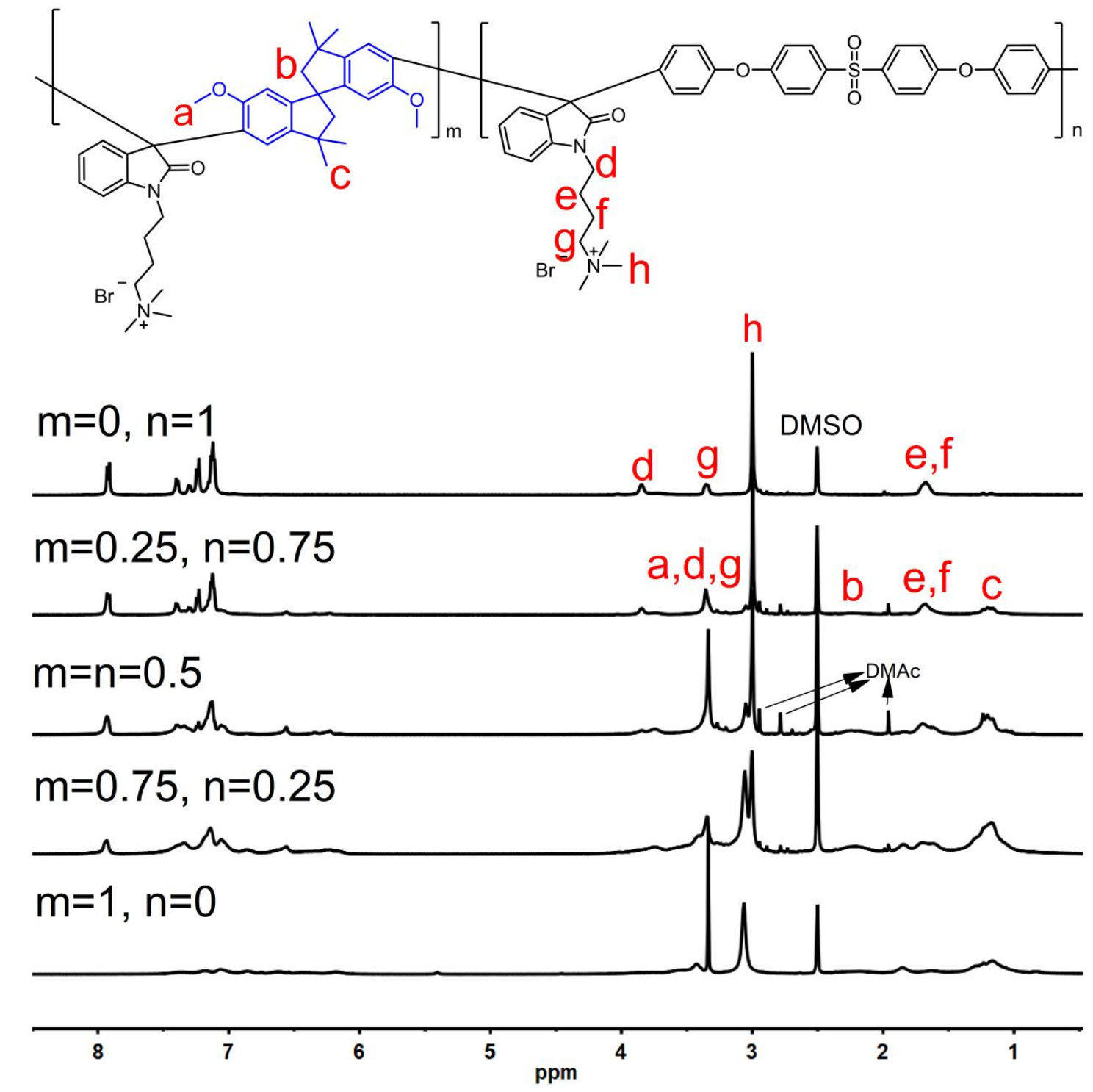

Figure S5. The ${ }^{1} \mathrm{H}$ NMR spectra of $\mathrm{QP}(\mathrm{SBI} / \mathrm{AES})-\mathrm{X}$.
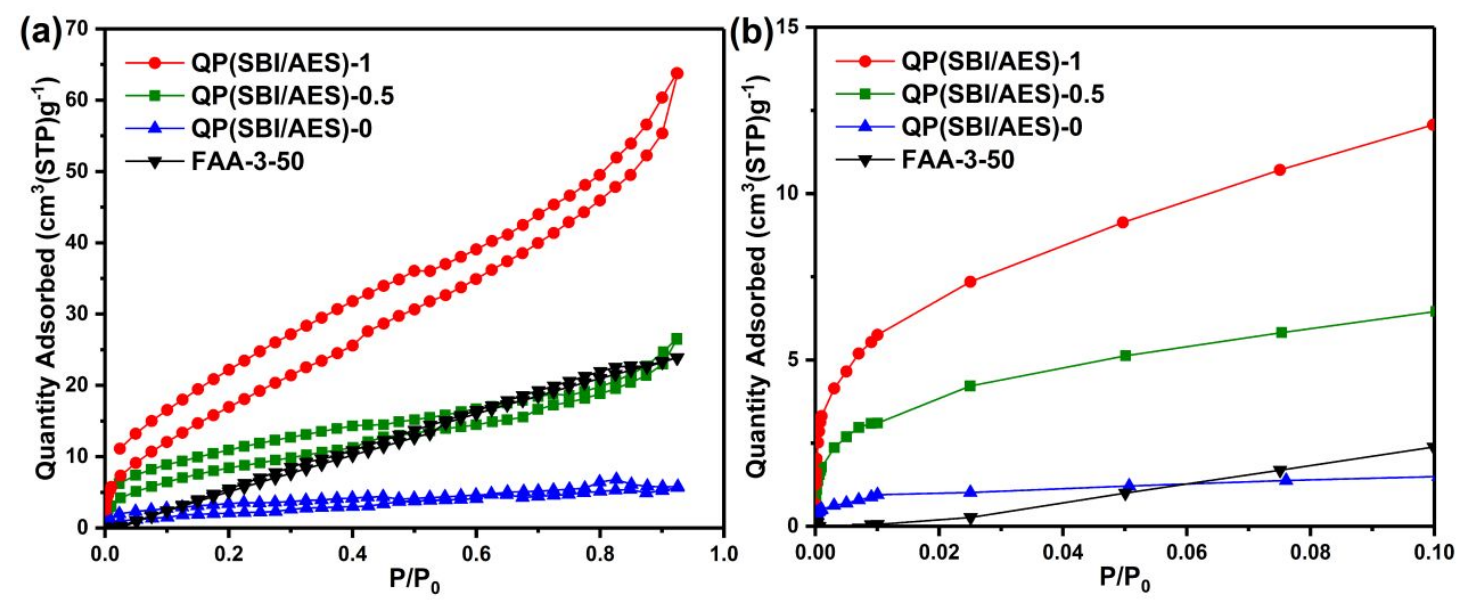
Figure S6. $\mathrm{N}_{2}$ adsorption isotherms of $\mathrm{QP}(\mathrm{SBI} / \mathrm{AES})-\mathrm{X}(\mathrm{X}=0,0.5,1)$ and FAA$3-50$ at $77 \mathrm{~K}$.

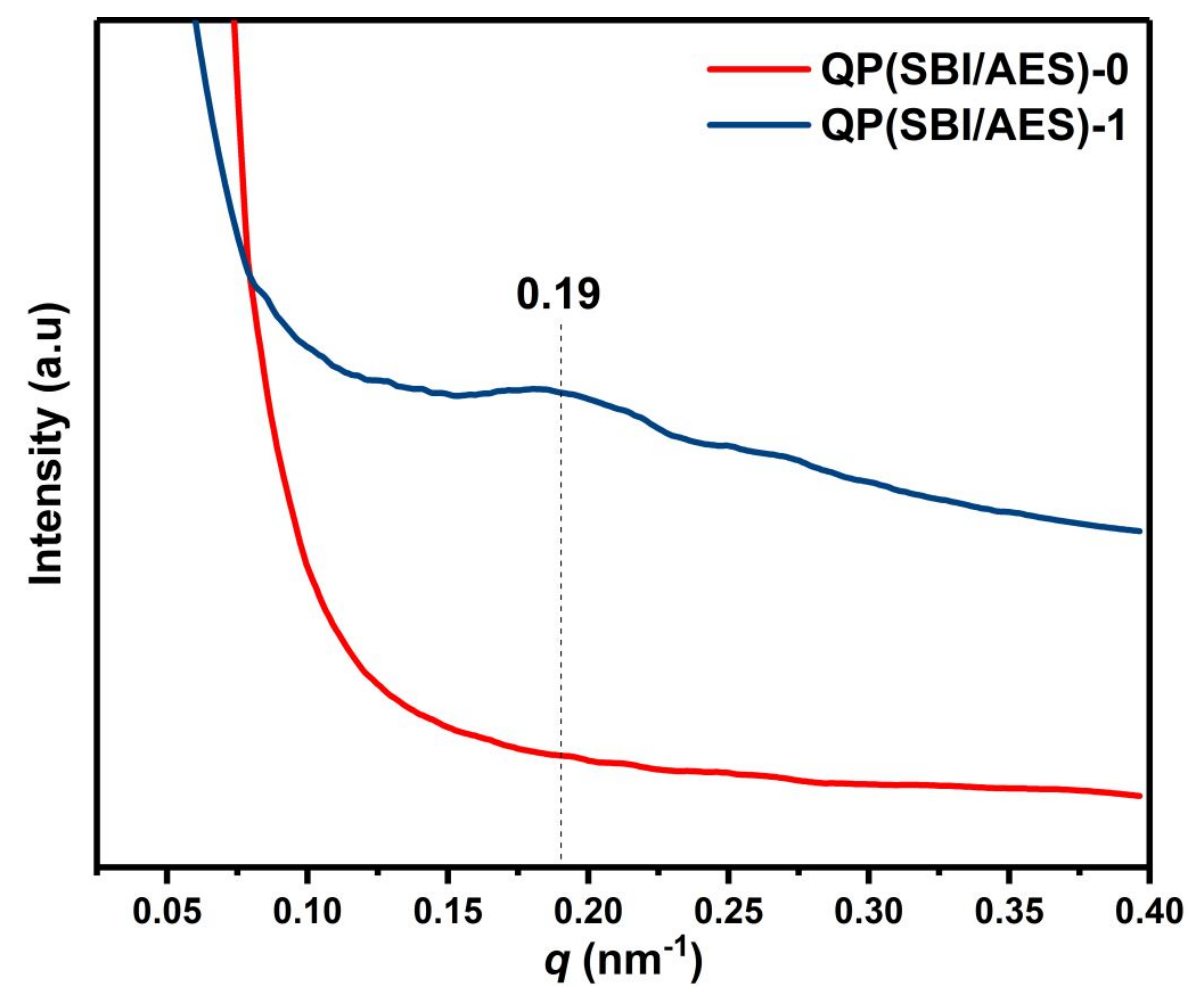

Figure S7. SAXS diagram of $Q P(S B I / A E S)-X(X=0,1)$. 

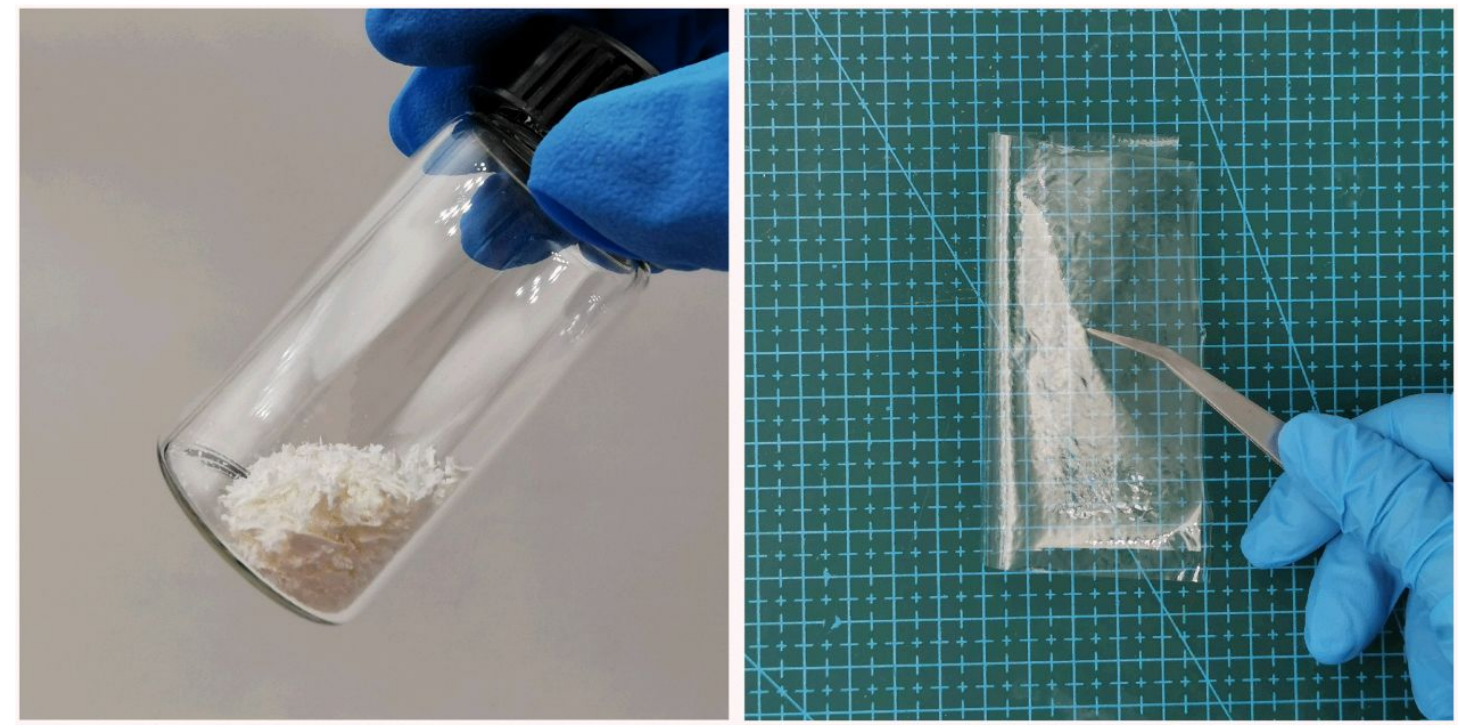

Figure S8. Photos of powder and dry membrane of $\mathrm{QP}(\mathrm{SBI} / \mathrm{AES})-0.5$ ionomer.

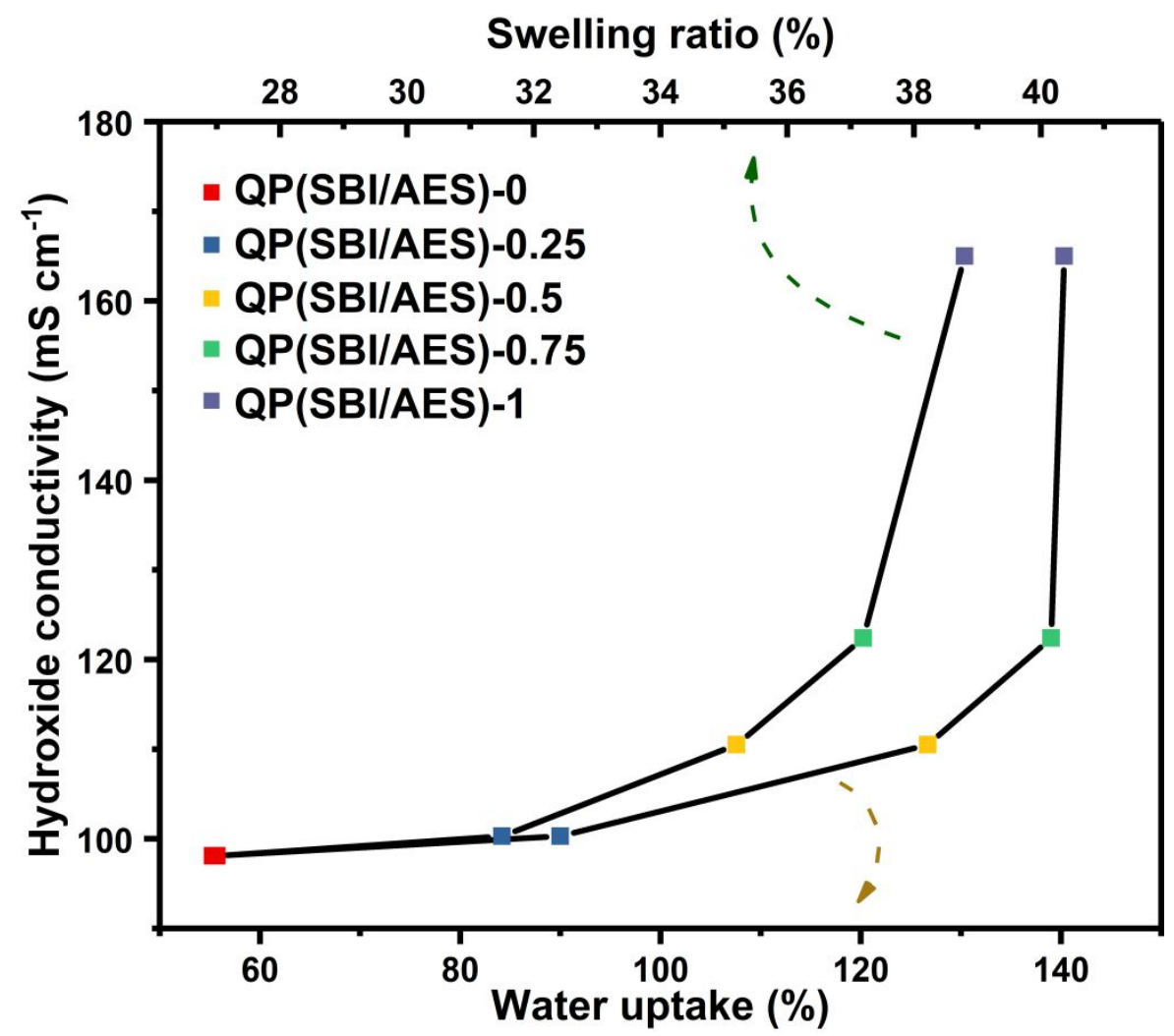

Figure S9. Hydroxide conductivity as a function of water uptake and swelling ratio at $80^{\circ} \mathrm{C}$. 


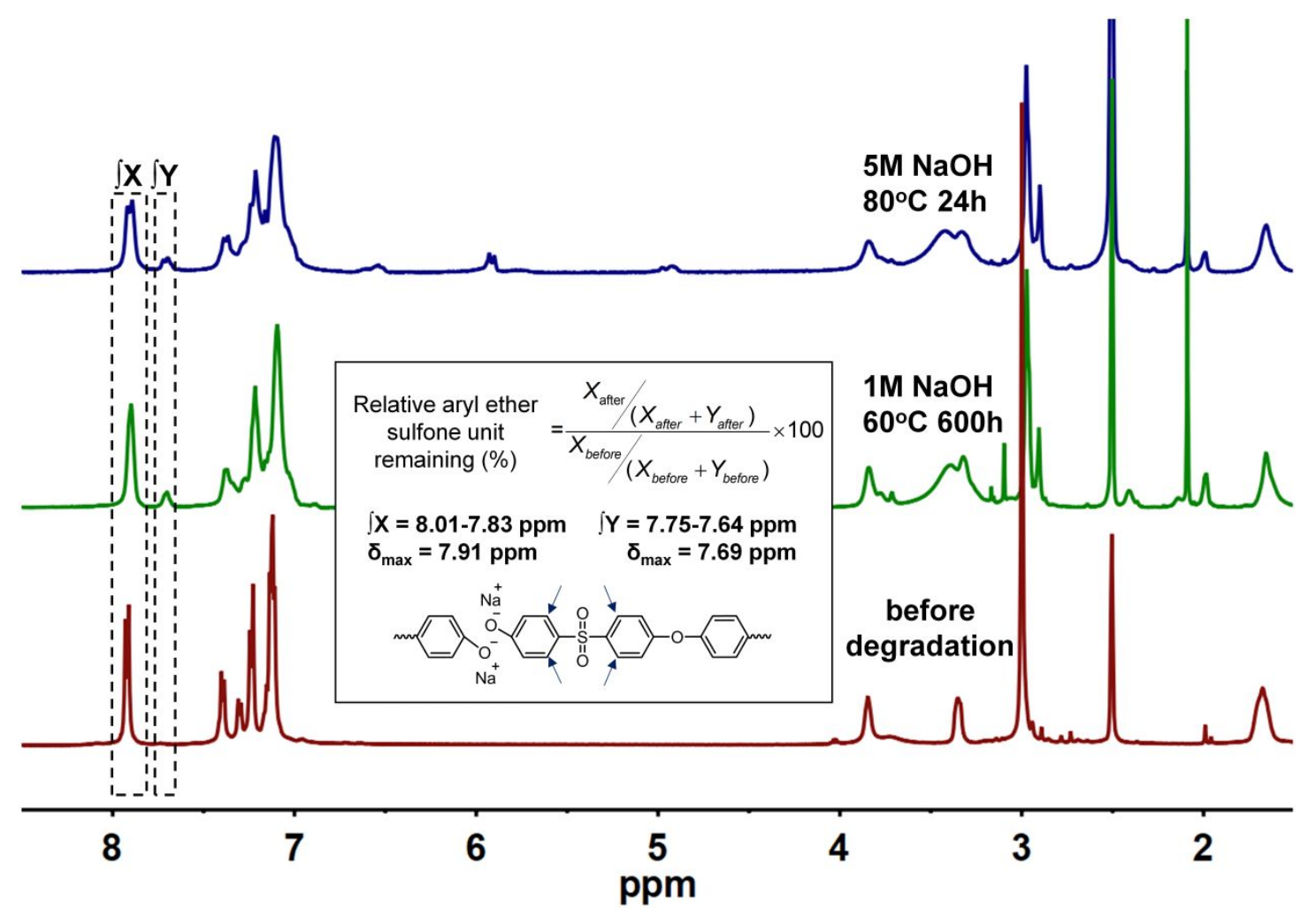

Figure S10. Degradation of QP(SBI/AES)-0 that has been immersed in $\mathrm{NaOH}$ aqueous solution is observed by ${ }^{1} \mathrm{H}$ NMR by comparison of integrals of the protons near the sulfone group before degradation $\left(\delta_{\max }=7.91 \mathrm{ppm}\right)$ and after degradation $\left(\delta_{\max }=7.69 \mathrm{ppm}\right)$. The spectra are individually normalised to the signal peak at $1.53-1.80 \mathrm{ppm}$. The same degradation of $\mathrm{QP}(\mathrm{SBI} / \mathrm{AES})-0.5$ is observed by ${ }^{1} \mathrm{H}$ NMR (Figure 6c).

Table S1. IECs of three membranes.

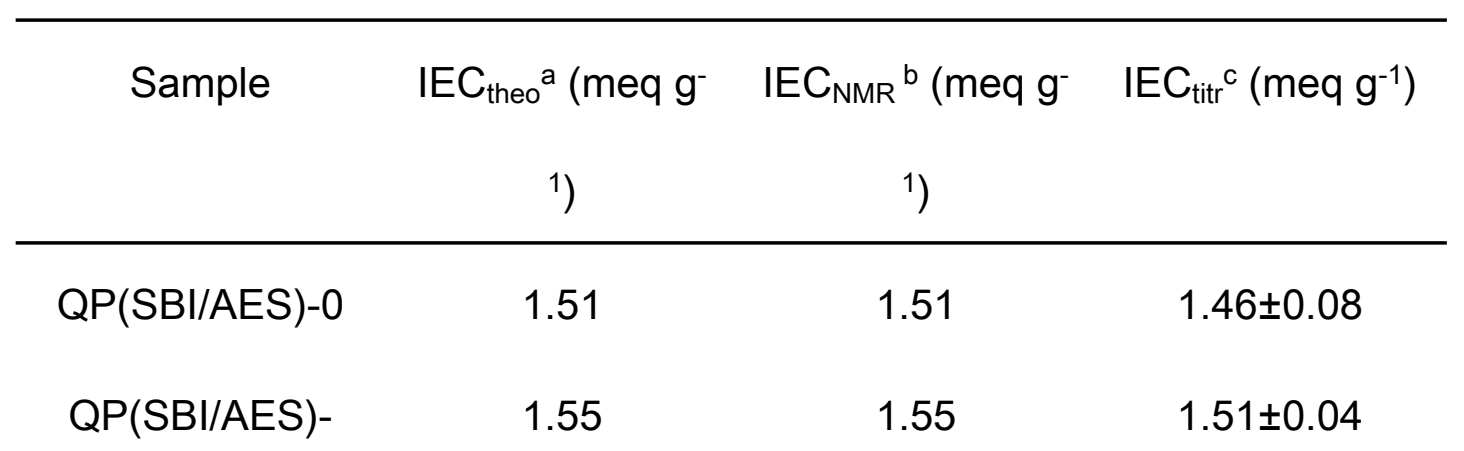



0.25
QP(SBI/AES)-0.5
1.59
1.58
$1.49 \pm 0.05$
QP(SBI/AES)-
1.63
1.61
$1.58 \pm 0.06$
0.75
QP(SBI/AES)-1
1.68
1.67
$1.61 \pm 0.07$
a) Theoretical value, calculated from the ratios of completely conversion.
b) Calculated value from the ${ }^{1} \mathrm{H}$ NMR (Fig. S1) by the ratio of hydrogen at 3.84 ppm and the $-\mathrm{CH}_{2}$ - at $2.98 \mathrm{ppm}$.
c) Experimental value measured by titration.

\section{Reference}

(1) Chen, W. F.; Lin, H. Y.; Dai, S. A. Generation and synthetic uses of stable

4-(2-isopropylidene)-phenol carbocation from bisphenol A. Org. Lett. 2004, 6 (14), 2341-2343, DOI: 10.1021/ol0493305

(2) Kendhale, A. M.; Gonnade, R.; Rajamohanan, P. R.; Hofmann, H. J.; Sanjayan, G. J. Foldamers with unusual structural architecture from spirobi(indane) building blocks. Chem. Commun. 2008, 22, 2541-2543, DOI: 10.1039/b800825f 